\title{
Severe infections and infection-related mortality in a large series of haploidentical hematopoietic stem cell transplantation with post-transplant cyclophosphamide
}

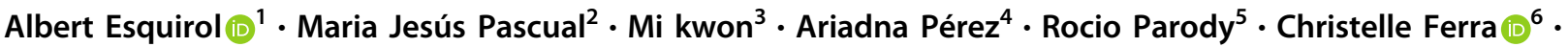 \\ Irene Garcia Cadenas $\mathbb{D}^{1} \cdot$ Beatriz Herruzo $^{2} \cdot$ Nieves Dorado $^{3} \cdot$ Rafael Hernani ${ }^{4} \cdot$ Isabel Sanchez-Ortega $^{5}$. \\ Anna Torrent ${ }^{6} \cdot$ Jorge Sierra $^{1}{ }^{1} \cdot$ Rodrigo Martino $\mathbb{D}^{1} \cdot$ Spanish Group for Hematopoietic Stem cell Transplantation \\ (GETH)
}

Received: 22 December 2020 / Revised: 10 April 2021 / Accepted: 22 April 2021 / Published online: 31 May 2021

(c) The Author(s), under exclusive licence to Springer Nature Limited 2021

\begin{abstract}
Severe infections and their attributable mortality are major complications in recipients of allogeneic hematopoietic stem cell transplantation (alloSCT). We herein report 236 adult patients who received haploSCT with PTCy. The median follow-up for survivors was 37 months. The overall incidence of bloodstream infections by gram-positive and gram-negative bacteria at 37 months was $51 \%$ and $46 \%$, respectively. The incidence of cytomegalovirus infection was $69 \%$, while Epstein Barr virus infections occurred in $10 \%$ of patients and hemorrhagic cystitis in $35 \%$ of cases. Invasive fungal infections occurred in $11 \%$ at 17 months. The 3-year incidence of infection-related mortality was $19 \%$. The median interval from transplant to IRM was 3 months (range 1-30), 53\% of IRM occurred >100 days post-haploSCT. Risk factors for IRM included age $>50$ years, lymphoid malignancy, and developing grade III-IV acute GvHD. Bacterial infections were the most common causes of IRM (51\%), mainly due to gram-negative bacilli BSI. In conclusion, severe infections are the most common causes of NRM after haploSCT with PTCy, with a reemergence of gram-negative bacilli as the most lethal pathogens. More studies focusing on the severe infections after haploSCT with PTCy and differences with other types of alloSCT in adults are clearly warranted.
\end{abstract}

Supplementary information The online version contains supplementary material available at https://doi.org/10.1038/s41409021-01328-4.

Albert Esquirol

aesquirol@santpau.cat

1 Hematology Department, Hospital de la Santa Creu i Sant Pau, IIB-Sant Pau and Jose Carreras Leukemia Research Institutes, Universitat Autonoma of Barcelona, Barcelona, Spain

2 Hematology Department, Hospital Regional Universitario, Malaga, Spain

3 Hematology Department, Hospital Gregorio Marañón, Madrid, Spain

4 Hematology Department, Hospital Clinico Universitario, Valencia, Spain

5 Hematology Department, Hospital de Bellvitge, Barcelona, Spain

6 Hematology Department, Hospital Germans Trias i Pujol, Badalona, Spain

\section{Introduction}

Over the last decade, several haploidentical hematopoietic stem cell transplantation (haploSCT) strategies have been developed to overcome HLA barriers [1-4], improving the high incidence of graft rejection and graft versus host disease associated with earlier haploSCT experiences. Currently, haploSCT has become a real alternative for patients lacking identical donor, mostly after the introduction of post-transplant cyclophosphamide [5-11].

Bloodstream infections (BSI) are the most common severe infections and are a major cause of mortality in patients undergoing allogeneic hematopoietic stem cell transplantation (alloSCT) [12], with an incidence ranging from 13 to $46 \%$ [13]. Many risk factors for BSI and severe infectious complications exist, such as prolonged severe neutropenia, myeloablative conditioning regimens, severe mucosal damage, use of broad-spectrum antibiotics; acute graft versus host disease, prolonged corticosteroids, and previous infectious history [13-16]. Moreover, delayed immune recovery, as seen with ex-vivo T cell-depleted alloSCT leads to high incidence 
of late infections in the haploSCT setting, as reported with the Perugia platforms [1, 17].

Post-transplant cyclophosphamide (PTCy) was a major milestone in the haploSCT setting. PTCy removes selectively alloreactive donor $\mathrm{T}$ cells that are proliferating in response to host alloantigen while preserving nonalloreactive donor $\mathrm{T}$ cells [18], with surprisingly fast quantitative immune reconstitution [4, 19]. Despite initial encouraging results with PTCy [20], infection-related mortality (IRM) is still the most common cause of mortality, and possibly higher than in alloSCT from HLA identical sibling donor (21\% vs. $13 \%, p=0.002)$ [11]. Likewise, IRM was higher in haploSCT without PTCy than a matched cohort of recipients of HLA identical sibling alloSCT $(26+$ $6 \%$ vs. $10+4 \%, p=0.04)$ [21].

Currently, despite several advances that have improved the outcomes after alloSCT, infectious complications remain a significant problem and a major cause of transplant failure.

In the present study, we describe the incidence of infections and causative pathogens in different post-SCT periods (pre-engraftment $[<31$ days $]$ (PE), early postengraftment [31-100 days] and late post-engraftment [>100 days]), the IRM and causative pathogens, as well as the overall transplant outcomes, in a large retrospective series of haploSCT with PTCy as graft versus host disease (GvHD).

\section{Patients and methods}

\section{Patients}

Two-hundred thirty-six adult consecutive patients were transplanted between November 2013 and November 2018 in six centers of the Spanish transplant group (Grupo Español de Transplante Hematopoyético [GETH]). All patients received a haploSCT using PTCy as GvHD prophylaxis [16] followed by calcineurin inhibitors, with or without mycophenolate mofetil (MMF). Transplants were done according to the local institutional protocols, and all patients signed informed consent. As a general rule, exclusion criteria were a poor performance status (ECOG $\geq$ 3 or Karnofsky score $<60 \%$ ), HIV infection, impaired cardiac or pulmonary functions, active viral hepatitis, and renal failure (creatinine $>$ x $1.5 \mathrm{ULN}$ ). The information was evaluated retrospectively for each patient.

\section{Conditioning regimens, Gvhd prophylaxis, and stem cell source}

The conditioning regimens were selected by each institution according to patients' characteristics and local protocols.
Myeloablative conditioning (MAC) regimens were fludarabine $\left(40 \mathrm{mg} / \mathrm{m}^{2} \mathrm{IV}\right)$ from day -6 to -3 and busulphan $(3.2 \mathrm{mg} / \mathrm{kg} \mathrm{IV})$ from day -6 to -3 (FluBu4) [22]; or thiotepa $(5 \mathrm{mg} / \mathrm{kg} \mathrm{IV})$ on days -7 and -6 , fludarabine (50 $\mathrm{mg} / \mathrm{m}^{2} \mathrm{IV}$ ) on days $-5,-4$, and -3 and busulphan $(1 \mathrm{mg} /$ $\mathrm{kg} / 6 \mathrm{~h}$ on days $-5,-4$, and -3 oral dose, or $3.2 \mathrm{mg} / \mathrm{kg} /$ day IV dose on the same days) (TBF) [23].

Reduced-intensity conditioning regimens (RIC) consisted of fludarabine $\left(30 \mathrm{mg} / \mathrm{m}^{2} \mathrm{IV}\right)$ from day -6 to -2 , cyclophosphamide ( $14.5 \mathrm{mg} / \mathrm{kg} \mathrm{IV})$ on days -6 and -5 and busulphan $(3.2 \mathrm{mg} / \mathrm{kg} \mathrm{IV})$ on days -4 and -3 (FluCyBu2). If the TBF platform was used as RIC, busulphan was reduced from three to 2 days (days -5 and -4 ).

All patients received PTCy $(50 \mathrm{mg} / \mathrm{kg}$ IV on days +3 and +4$)$ as GvHD prophylaxis followed by cyclosporine combined with MMF or tacrolimus alone since day +5 [22, 23]. Intravenous MESNA was given at a dose of 10 $\mathrm{mg} / \mathrm{kg} / 6 \mathrm{~h}$ on days +3 and +4 (total daily dose of $40 \mathrm{mg} /$ $\mathrm{kg}$ ) as hemorrhagic cystitis (HC) prevention.

Each institution chose to use either peripheral blood (PB) or bone marrow (BM) as the stem cell (SC) source. The target dose of CD34+ cells/ $\mathrm{kg}$ of recipient weight to be infused was $5 \times 10^{6} / \mathrm{kg}$ (range $4-6 \times 10^{6} / \mathrm{kg}$ ) in recipients of PBSC transplantation (PBSCT), while the target dose total nucleated cells (TNC)/kg recipient weight in BM recipients was $3 \times 10^{8} / \mathrm{kg}$.

The centers selected the haploidentical donor based on availability and their preference among the first-degree relatives. Donor-specific anti-HLA antibodies (DSA) were studied in all patients; a local protocol of desensitisation was in place in case of inevitable high DSA titers were found (details not shown).

\section{Definitions and supportive care}

The definitions used in the current study are also shown in detail in the Supplementary online material.

In general, any bacterial, viral, or invasive fungal infection (IFI) requiring intravenous treatment or hospitalization was considered a severe infectious episode. In the case of common bacterial skin contaminants (mostly coagulase-negative staphylococci), a bloodstream infection (BSI) was diagnosed only if $\geq 2$ consecutive blood cultures were positive for the same species. Infection data were collected retrospectively until the patient's death or last follow-up, using standardized definitions of severe infections after SCT based on the most recent guidelines (https://www.ebmt.org/working-parties/ infectious-diseases-working-party-idwp). Common respiratory virus infections and virus-related hemorrhagic cystitis were also included in the study. Cytomegalovirus (CMV) infection was defined as the presence of at least 2 consecutive (within a minimum interval of $48 \mathrm{~h}$ ) positive results of a 
polymerase chain reaction (PCR) test for $\mathrm{CMV}$ in peripheral blood (PB) with a viral load $>1000 \mathrm{IU} / \mathrm{mL}$, while CMV disease was defined as the demonstration of CMV in biopsy or autopsy specimens from clinically involved visceral sites by culture and/or histology or if CMV was detected in samples from clinically-defined sites of disease. Epstein-Bar virus (EBV) infection was defined as the presence of at least 2 consecutive (within a minimum interval of 7 days) positive results of a PCR test for EBV in peripheral blood (PB) with a viral load $>1000$ e.g.c. $/ \mathrm{mL}$, with or without evidence of EBVrelated post-transplant lymphoproliferative disease (EBVPTLD). Human herpes virus 6 disease was defined as the presence of positive PCR test in cerebrospinal fluid in patients with neurological symptoms (encephalitis), and a positive intestinal biopsy (colitis).

All patients were nursed in HEPA-filtered rooms. Antimicrobial prophylaxis was given following institutional policies, but which can be summarized as follows. Bacterial prophylaxis consisted of ciprofloxacin or levofloxacin during neutropenia or until the start of broad-spectrum antibiotics. Antifungal prophylaxis was administered according to the protocols at each site, with either fluconazole and a pre-emptive strategy; or a mold-active antifungal agent (posaconazole, voriconazole or other systemic antifungal drugs) until engraftment or whenever the patient was given steroids for the treatment of GVHD. Prophylaxis against Pneumocystis jirovecci consisted of cotrimoxazole until day -2 and then was restarted after engraftment. Pentamidine was used if cotrimoxazole was contraindicated. For prevention of Cytomegalovirus (CMV)-related disease, all institutions followed a preemptive approach with polymerase chain reaction (PCR) monitoring; the treatment started with positive PCR consisted of ganciclovir or foscarnet if severe neutropenia or ganciclovir toxicities. Acyclovir was recommended for a minimum of 1 -year postHSCT or until immunosuppressive therapy was stopped. Galactomannan testing and CMV PCR analysis were performed twice weekly, and Epstein-Barr virus (EBV) PCR analysis was performed weekly until day +100 , in case of EBV infection, rituximab was used. Intravenous immunoglobulin (IVIG) replacement was recommended whenever the total IgG blood level was $<400 \mathrm{mg} / \mathrm{dL}$, especially if the patient had prior infection by encapsulated bacteria or were considered to be at high risk of humoral immunodeficiency-linked opportunistic infections. All centers started the practice of vaccination after 6th months unless patients had severe active GvHD or were receiving IVIG therapy

\section{Statistical analyses}

SPSS statistics (IBM SPSS Statistics 21) and R studio programs (R studio, Boston, MA) were used for statistical analyses. Overall survival (OS) was defined as the time from day 0 to date of death by any cause, and progressionfree survival (PFS) was the time from day 0 to disease progression or death. The Kaplan Meier method was used for estimating the actuarial PFS and OS, and the log rang test was used to study the univariate impact of any given variable on OS and PFS. The cumulative incidence (CI) estimate with competing risk(s) analysis was used to calculate the incidence of acute and chronic GVHD, nonrelapse mortality (NRM), relapse, and infection-related mortality (IRM). The competing risk for NRM was relapse, while for relapse it was NRM. Competing risks for acute and chronic GvHD were disease relapse and NRM up to 100 days after stem cell infusion for acute GvHD and until the last follow up for chronic GvHD. Competing risk for IRM was NRM not due to infection or relapse. Competing risk of infection during a period of infectious risk was NRM or relapse during the time period. Gray test was used to study the impact of any given variable on a CI. We used landmark studies to find the incidence of infections in different time points, and thus time-dependent variables which occurred before each landmark point were included as binary variables. If the patients were alive at every time point they were categorized in two groups (with or without infection). Patients who died before the time of any given landmark point were excluded from the next time point. The time points: <day 31 (pre-engraftment), between days 31-100 (early post engraftment) and > day 100 (late postengraftment) were used for infections by GPB, GNB, and conventional respiratory virus infection. For CMV and EBV, hemorrhagic cystitis and fungal infections, the time points selected were $<31$ and $\geq 31$ days. COX regression analysis was used for multivariate analysis with documentation of proportional hazards over time for each outcome and covariate analyzed. In addition, variables or events that occurred post-transplant but before a given landmark point were analyzed as possible risk factors for IRM, NRM and relapse in the framework of Cox models as time-dependent covariates (for instance, occurrence of aGVHD), switching from absent to present at the moment of occurrence of each covariate/event.

\section{Results}

\section{Patient and donor characteristics}

Patient and donor characteristics are shown in Table 1. The median follow up of survivors was 37 months (range 12-82). The median patient age was 50 years (range $17-71$ ), and $61 \%$ were male. Seventy-six patients (32\%) had acute myeloid leukemia (AML), which was the most common underlying disease, followed by lymphoma in 70 
Table 1 Patient and donors' characteristics.

\begin{tabular}{|c|c|}
\hline Number of cases & 236 \\
\hline Patients' characteristics & $N(\%)$ \\
\hline Median age [range] & $50(17-71)$ \\
\hline Age $\geq 50$ years $/ \geq 60$ years & $115(49 \%) / 57(24 \%)$ \\
\hline Male and female sex & $144(61 \%) / 92(39 \%)$ \\
\hline Female donor to male recipient & $64(27 \%)$ \\
\hline \multicolumn{2}{|l|}{ Underlying disease } \\
\hline AML & $76(32 \%)$ \\
\hline MDS & $39(17 \%)$ \\
\hline ALL & $22(9 \%)$ \\
\hline Non-Hodgkin's lymphoma & $39(17 \%)$ \\
\hline Hodgkin disease & $31(13 \%)$ \\
\hline CLL & $8(3 \%)$ \\
\hline CML or other MPS & $12(5 \%)$ \\
\hline Multiple myeloma & $5(2 \%)$ \\
\hline Biphenotypic acute leukemia & $2(1 \%)$ \\
\hline Aplasia & 1 \\
\hline Prolymphocytic leukemia & 1 \\
\hline \multicolumn{2}{|l|}{ Response at transplant } \\
\hline Complete remission (first and second) & $130(55 \%)$ \\
\hline Third complete remission & $16(7 \%)$ \\
\hline Partial remission & $33(14 \%)$ \\
\hline Stable disease & $17(7 \%)$ \\
\hline Progression or refractory disease & $33(14 \%)$ \\
\hline Induction chemotherapy aplasia & $5(2 \%)$ \\
\hline Primary graft failure & $2(1 \%)$ \\
\hline \multicolumn{2}{|l|}{ Refined Disease Risk Index (rDRI) } \\
\hline Low rDRI & $29(12 \%)$ \\
\hline Intermediate rDRI & $125(54 \%)$ \\
\hline High rDRI & $72(31 \%)$ \\
\hline Very High rDRI & $7(3 \%)$ \\
\hline Prior HSCT, num. (\%) & $77(33 \%)$ \\
\hline Previous aloSCT & $27(11 \%)$ \\
\hline \multicolumn{2}{|l|}{ Conditioning regimen } \\
\hline FluBu & $23(10 \%)$ \\
\hline FluBuCy & $87(37 \%)$ \\
\hline TBF & $121(51 \%)$ \\
\hline Other (FluCyTBI, FluATG) & $5(2 \%)$ \\
\hline \multicolumn{2}{|l|}{ Conditioning intensity } \\
\hline Myeloablative & $75(32 \%)$ \\
\hline Reduced intensity & $161(68 \%)$ \\
\hline \multicolumn{2}{|l|}{ Stem cell source } \\
\hline Peripheral blood stem cells & $191(81 \%)$ \\
\hline Bone marrow & $45(19 \%)$ \\
\hline \multicolumn{2}{|l|}{ GvHD prophylaxis following PTCy } \\
\hline Cyclosporine with MMF & $115(49 \%)$ \\
\hline \multirow[t]{2}{*}{ Tacrolimus } & $121(51 \%)$ \\
\hline & $5,4(1.95-11.42)$ \\
\hline
\end{tabular}

Table 1 (continued)

CD34 + cells infused $(\times 106 / \mathrm{kg})$

(median, range)

Median follow-up in survivors, months 37 [1-82]

(range)

Donors' characteristics

Male and female sex

$132(56 \%) / 104(44 \%)$

Donor relationship with patient

Mother/Father

$13(6 \%) / 23(10 \%)$

Son/Daughter $61(26 \%) / 41(17 \%)$

Brother/Sister $55(23 \%) / 39(16 \%)$

Other donors $4(2 \%)$

Donor and recipient CMV IgG combination
$\mathrm{D}+/ \mathrm{R}+-$
$112(48 \%)$
$\mathrm{D}-/ \mathrm{R}+$
$69(29 \%)$
$\mathrm{D}+/ \mathrm{R}-$
$29(12 \%)$
$\mathrm{D}-/ \mathrm{R}-$
$25(11 \%)$

$A M L$ acute myeloid $M D S$ Myelodysplastic syndrome, $A L L$ acute lymphoblastic leukemia, $C L L$ chronic lymphocytic leukemia, $C M L$ chronic myeloid leukemia, MPN myeloproliferative neoplasm, HSCT hematopoietic stem cell transplantation, AlloSCT allogeneic stem cell transplantation, $F l u B u$ fludarabine-busulfan, $F l u B u B y$ fludarabinebusulfan-cyclophosphamide, TBF thiotepa-fludarabine-busulfan, FluCyTBI fludarabine-cyclophosphamide-total body irradiation, FluATG fludarabine-ATG, $G v H D$ graft versus host disease, PTCy posttransplantation cyclophosphamide, $M M F$ mycophenolate mofetil, $C M V$ cytomegalovirus, $D$ donor, $R$ recipient.

patients (30\%). Twenty-seven patients (11\%) had failed a first alloSCT.

One hundred thirty-five patients (57\%) were in early disease phase at transplant (first and second CR; and postinduction aplasia), although 79 patients (34\%) had a high or very high refined Disease Risk Index (rDRI) [24]. MAC regimen was used in $32 \%$ of the transplants (75 patients).

One hundred thirty-two donors (56\%) were male, and the most common donors used was a son $(26 \%)$ or a daughter (17\%). An IgG seropositive CMV donor for a seropositive patient was the most frequent combination in 112 donorpatient CMV serostatus (48\%), only $23 \%$ of patients were CMV seronegative.

\section{Hematological recovery; acute and chronic GVHD}

One hundred ninety-one patients $(81 \%)$ received PBSC. The median number of CD34 + in PBSCT was $5.4 \times 10^{6} / \mathrm{kg}$ (range 1.95-11.42), while the TNC infused in BM recipients was $3.5 \times 10^{6} / \mathrm{kg}$ (range $0.84-19.6$ ). The CI of neutrophil and platelet recovery was $94 \%$ (95\% CI, 91-97\%) and 90\% (95\% CI, 86-94\%), respectively. Six patients (2.5\%) had primary graft failure. Nine patients died early (before day +21 ) without engraftment (with a severe infection as primary cause of death), including 5/27 (19\%) previously allotransplanted patients. 
In the 221 remaining patients, the median time to neutrophil $\left(>0.5 \times 10^{9} / \mathrm{L}\right)$ and platelet $\left(>20 \times 10^{9} / \mathrm{L}\right)$ recovery was 18 days (range 9-49) and 26 days (range 10-156), respectively.

The CI of grade II-IV and III-VI acute GvHD at day +100 was $31 \%$ (95\% C.I., 25-37\%) (79 patients) and $11 \%$ (95\% C.I., 7-15\%) (30 patients), respectively; 5 patients died as a consequence of steroid-refractory grade III-IV acute GvHD.

The CI of limited, moderate, and severe chronic GvHD at 37 months was $16 \%$ (95\% C.I., 12-22\%), 8\% (95\% C.I., 5-11\%), and 7\% (95\% C.I., 4-10\%), respectively. Four patients died due to severe chronic GvHD.

\section{Transplant outcomes}

The OS at 12 and 37 months was 64\% (95\% C.I., 61-67\%) and $50 \%$ (95\% C.I., 46-54\%), respectively, and the PFS was $57 \%$ (95\% C.I., 54-60\%) and 47\% (95\% C.I., 44-50\%), respectively. Several variables had an independent impact on these outcomes in multivariate analysis (MVA); first and second CR at transplant, patients $\leq 50$ years and grade III-IV acute GvHD for OS; and first and second CR at transplant, rDRI and patient age $\leq 50$ years for PFS [Table 2].

The CI incidence of relapse at 12 and 37 months was $17 \%$ (95\% CI, 12-22\%) and 21\% (95\% CI, 16-26\%), respectively. Variables that impacted on relapse in MVA were the disease response at transplant (first and second CR vs. other responses) and low-intermediate rDRI [Table 2]. Forty patients (17\%) died owing to relapse.

The CI of non-relapse mortality (NRM) was $26 \%(95 \%$ CI, $21-31 \%)$ and $31 \%(95 \% \mathrm{CI}, 25-37 \%)$ at 12 and 37 months. Prior alloSCT, age $\geq 50$ years and grade III-IV acute GVHD were risk factors in the MVA [Table 2]. The main cause of NRM was an IRM $(58 \%$ of NRM, 43 patients) [Table 3].

\section{Infection-related mortality and severe infectious complications}

The CI of IRM at 12 months was $17 \%$ (95\% C.I., 12-22\%) and $19 \%$ (95\% C.I., 14-24\%) at 37 months, and the variables with an independent impact in the MVA analysis were age $\geq 50$ years, lymphoid malignancy as underlying disease (vs. myeloid), and development of grade III-IV acute GvHD [Table 2].

Six hundred twenty-three severe infectious episodes were reported in the 236 patients, with 2.6 infections per patient (range 0-8). Only 17 patients (7\%) did not develop any severe infections. In addition, 14\% (32 patients) developed a clinically defined severe infection without microbiological documentation, and $10 \%$ required ICU admission due to a severe infection.
Fifty-six percent of patients developed at least one bacterial infection (19\% of patients had gram-positive bacterial infections, $15 \%$ had gram-negative bacterial infections, and $22 \%$ had both types of bacterial infections). CMV infection (CMV-I) was found in $69 \%$ of patients, and $52 \%$ had at least one non-CMV viral infection. An invasive fungal infection (IFI) occurred in 41 patients (17\%), including $10 \%$ of possible, probable, or proven invasive aspergillosis. Specific pathogens involved, their distribution post-transplant, and the median time to onset of the major types of infections per time period are shown in detail in Table 4.

There were 43 cases of IRM; the median interval from transplant to IRM was 3 months (range 10-927); eight (19\%) died during the PE period, 12 patients $(28 \%)$ developed a lethal infectious complication in the early post-engraftment period, and 23 patients $(53 \%)$ died beyond +100 days. In $51 \%$ (22 patients), the cause of IRM was bacterial, $16 \%$ (7 patients) developed a lethal viral infection, 5\% (2 patients) an IFI, and two patients had a mixed bacterial and fungal infection. In $23 \%$ of clinically documented IRM no microbiological documentation was reported. The documented primary causes of IRM and NRM are shown in Table 3.

All IS was discontinued in 161 of patients $(68 \%)$ at a median time post-transplant of 199 days (range 1-37 months), and seven of these patients died as a consequence of infection (4.3\% IRM). Six of these seven patients died after day +100 .

In 75 patients $(32 \%)$ discontinuation of IS therapy had not been possible at last follow-up. Thirty-three patients died before 100 days, and the main causes of death were infection (19 patients, $25 \%$ IRM), while of the 42 patients who received IS drug/s after day $+100,17$ died due to infections (40\% IRM) and 12 patients were still alive at last follow-up more than 1 year post-transplant.

\section{Bacterial infections}

The incidence of PE $(<$ day +31$)$, early post-engraftment $($ day +31 to +100$)$ and late post-engraftment $(>$ day +100$)$ infections by gram-positive bacteria (GPB) was $20 \%(95 \%$ C.I., $15-25 \%$ ), $11 \%$ (95\% C.I., 7-15\%) and $20 \%$ (95\% C.I., 14-26\%), respectively, with an overall incidence of $51 \%$. The median time of infection was 10 days (range 0-28), 72 days (range 32-97), and 7 months (range 3-29), respectively.

One hundred twenty-two infection episodes by GPB were reported (shown in detail in Table 4) of which $46 \%$ (56 episodes) occurred in the PE period. Staphylococcus spp. and Enterococcus spp. were the most frequent GPB. Six of seven patients with Streptococcus spp. infection in the late post-engraftment period were caused by Streptococcus pneumoniae. Only eight GPB $(6 \%)$ presented antibiotic resistance (Methicillin-resistant Staphylococcus 


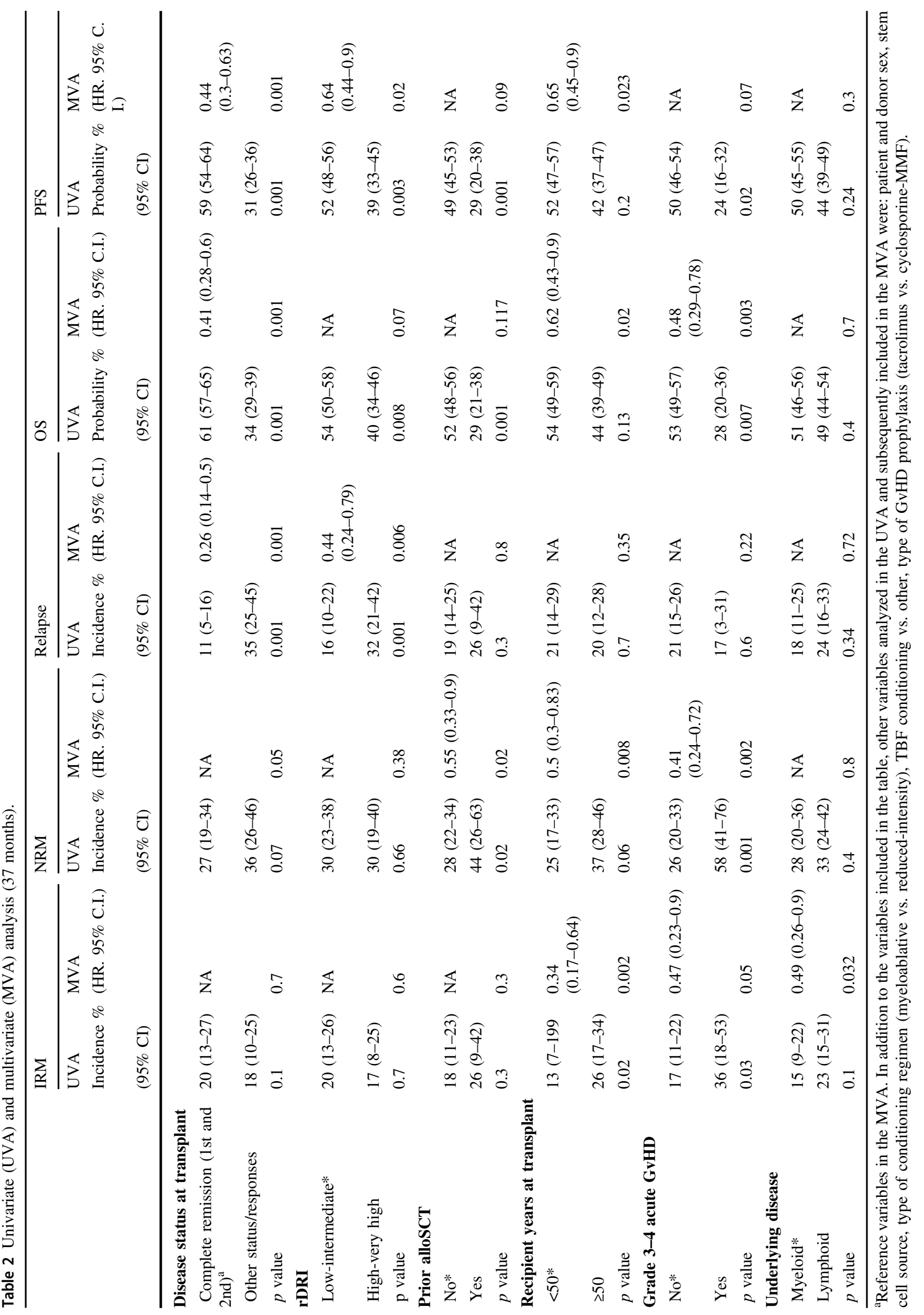


Table 3 Causes of nonrelapse mortality.

\begin{tabular}{|c|c|c|c|}
\hline & $\begin{array}{l}\text { Pre-engraftment } \\
(<31 \text { days })\end{array}$ & $\begin{array}{l}\text { Early post-engraftment } \\
\text { (31-100 days) }\end{array}$ & $\begin{array}{l}\text { Late post-engraftment } \\
\text { (>100 days) }\end{array}$ \\
\hline Causes of IRM N $43(58 \%)$ & $8(19)$ & $12(28)$ & $23(53)$ \\
\hline Gram positive bacterial (N 7) & 2 & 3 & 2 \\
\hline Enterococcus faecium & 2 & & \\
\hline Enterococcus faecalis & & 1 & 1 \\
\hline Streptococcus mitis & & 1 & \\
\hline Stretococcus pneumoniae & & & 1 \\
\hline $\begin{array}{l}\text { Methicillin resistance } \\
\text { Staphylococcus aureus }\end{array}$ & & 1 & \\
\hline Gram negative bacterial (N 13) & 2 & 2 & 9 \\
\hline Escherichia coli & & & 1 \\
\hline ESBL Escherichia coli & 1 & & \\
\hline Pseudomonas aeruginosa & & & 1 \\
\hline $\begin{array}{l}\text { Multidrug resistance } \\
\text { Pseudomonas aeruginosa }\end{array}$ & 1 & 1 & 1 \\
\hline Klebsiella pneumoniae & & & 2 \\
\hline $\begin{array}{l}\text { ESBL or KPC Klebsiella } \\
\text { pneumoniae }\end{array}$ & & 1 & 1 \\
\hline Acinetobacter baumannii & & & 1 \\
\hline Stenotrophomonas maltophila & & & 1 \\
\hline Serratia marcescens & & & 1 \\
\hline Viral infection (N 7) & 1 & 3 & 3 \\
\hline$C M V$ disease (pneumonitis) & 1 & 2 & 1 \\
\hline Metapneumovirus & & 1 & \\
\hline Human herpes 6 virus & & & 1 \\
\hline$E B V-P T L D$ & & & 1 \\
\hline Invasive fungal infection (N 2) & 1 & & 1 \\
\hline Probable IFI & 1 & & 1 \\
\hline $\begin{array}{l}\text { Gram negative bacterial }+ \\
\text { fungal infection }(\mathbf{N} 2)\end{array}$ & & 1 & 1 \\
\hline $\begin{array}{l}\text { Gram negative and positive } \\
\text { bacterial (N 2) }\end{array}$ & & & 2 \\
\hline $\begin{array}{l}\text { Without positive } \\
\text { microbiological (N 10) }\end{array}$ & 2 & 3 & 5 \\
\hline $\begin{array}{l}\text { Other causes of NRM N } 26 \\
(35 \%)\end{array}$ & $4(15)$ & 9 (35) & $13(50)$ \\
\hline Graft rejection & 1 & 2 & 2 \\
\hline Sinusoidal obstruction syndrome & 2 & 1 & \\
\hline Idiophatic encephalitis & & 2 & 1 \\
\hline Refractory acute GvHD & & 2 & 3 \\
\hline Chronic GvHD & & & 4 \\
\hline Other NRM causes & 1 & 2 & 3 \\
\hline Secondary neoplasms N 5 (7\%) & & & 5 \\
\hline
\end{tabular}

Other NRM causes: non-infectious endocarditis (x1), adult respiratory distress syndrome (x1), refractory bleeding (x1), alveolar refractory bleeding (x2) and sudden death (x1).

$N R M$ non relapse mortality, IRM infection-related mortality, ESBL extended-spectrum beta-lactamase, $C M V$ cytomegalovirus, $P T-L P D$ posttransplant lymphoproliferative disorder, $E B V$ Epstein-Barr virus, $I F I$ invasive fungal infection, $P T L D$ post-transplant lymphoproliferative disease. 
Table 4 Microbiological etiology in time of onset.

\begin{tabular}{|c|c|c|c|}
\hline & $\begin{array}{l}\text { Pre-engraftment } \\
(<31 \text { days })\end{array}$ & $\begin{array}{l}\text { Early post-engraftment } \\
\text { (31-100 days) }\end{array}$ & $\begin{array}{l}\text { Late post-engraftment } \\
(>100 \text { days) }\end{array}$ \\
\hline $\begin{array}{l}\text { Total infectious episodes } N=623 \\
(100 \%)\end{array}$ & $205(33)$ & $214(34)$ & $204(33)$ \\
\hline $\begin{array}{l}\text { Gram positive bacteria } N=122 \\
(20 \%)\end{array}$ & $56(46)$ & $25(20)$ & $41(34)$ \\
\hline $\begin{array}{l}\text { Median time of GPB infections } \\
\text { days or months (range) }\end{array}$ & 10 days $(0-28)$ & 72 days (32-97) & 7 months $(3-29)$ \\
\hline Staphylococcus spp & 31 & 13 & 22 \\
\hline Enterococcus spp & 17 & 10 & 10 \\
\hline Streptococcus spp & 5 & & 7 \\
\hline Corynebacterium spp & 2 & & \\
\hline Bacillus spp & 1 & & \\
\hline Listeria monocytogenes & & 2 & 1 \\
\hline Nocardia spp & & & 1 \\
\hline $\begin{array}{l}\text { Gram } N=\text { egative bacterial } \\
\mathbf{N}=107(17 \%)\end{array}$ & $23(21)$ & $34(32)$ & $50(47)$ \\
\hline $\begin{array}{l}\text { Median time of GNB infections } \\
\text { days or months (range) }\end{array}$ & 13 days $(0-28)$ & 51 days (35-97) & 7 months $(3-33)$ \\
\hline Escherichia coli & 10 & 8 & 17 \\
\hline Pseudomonas aeruginosa & 7 & 10 & 14 \\
\hline Klebsiella pneumoniae & 2 & 7 & 8 \\
\hline Stenotrophomonas maltophilia & 3 & 1 & 3 \\
\hline Serratia marcescens & 1 & 2 & 1 \\
\hline Enterobacter cloacae & & 5 & 1 \\
\hline Haemophilus influenzae & & & 4 \\
\hline Veillonella spp & & 1 & \\
\hline Acinetobacter baumannii & & & 1 \\
\hline Leptotricia trevisanii & & & 1 \\
\hline Clostridium difficile $N=17$ (3\%) & $10(59)$ & $5(29)$ & $2(12)$ \\
\hline $\begin{array}{l}\text { Median time of CD infection days } \\
\text { or months (range) }\end{array}$ & 5 days $(2-20)$ & 42 days $(32-47)$ & - \\
\hline \multicolumn{4}{|l|}{ Viral infections } \\
\hline Cytomegalovirus $N=155(25 \%)$ & $58(38)$ & $84(54)$ & $13(8)$ \\
\hline $\begin{array}{l}\text { Median time of CMV infections } \\
\text { days or months (range) }\end{array}$ & 21 days $(0-30)$ & 42 days $(31-100)$ & 5 months $(3-31)$ \\
\hline Reactivation & 54 & 84 & 12 \\
\hline Disease & 4 & - & 1 \\
\hline Epstein-Barr Virus $N=23(4 \%)$ & & $5(22)$ & $18(72)$ \\
\hline $\begin{array}{l}\text { Median time of EBV infections } \\
\text { days or months (range) }\end{array}$ & - & 85 days (69-98) & 5 months $(3-20)$ \\
\hline Reactivation & - & 5 & 16 \\
\hline EBV-PTLD & & & 2 \\
\hline $\begin{array}{l}\text { Hemorrhagic cystitis } N=77 \\
(12 \%)\end{array}$ & $32(42)$ & $37(48)$ & $8(10)$ \\
\hline $\begin{array}{l}\text { Median time of } \mathrm{HC} \text { infection days } \\
\text { or months (range) }\end{array}$ & 14 days $(0-30)$ & 45 days $(31-98)$ & 4 months $(3-7)$ \\
\hline BK Poliomavirus-related & 20 & 31 & 3 \\
\hline Adenovirus & 1 & 3 & 1 \\
\hline Without viral infection & 11 & 3 & 4 \\
\hline
\end{tabular}


Table 4 (continued)

\begin{tabular}{|c|c|c|c|}
\hline & $\begin{array}{l}\text { Pre-engraftment } \\
\text { ( }<31 \text { days })\end{array}$ & $\begin{array}{l}\text { Early post-engraftment } \\
\text { (31-100 days) }\end{array}$ & $\begin{array}{l}\text { Late post-engraftment } \\
(>100 \text { days) }\end{array}$ \\
\hline $\begin{array}{l}\text { Upper respiratory tract viral } \\
\text { infections } N=34(5 \%)\end{array}$ & $2(6)$ & $10(29)$ & $22(65)$ \\
\hline $\begin{array}{l}\text { Median time of URT infections } \\
\text { days or months (range) }\end{array}$ & - & 70 days (63-84) & 10 months $(4-42)$ \\
\hline Influenza virus & & 5 & 10 \\
\hline Respiratory syncytial virus & & 5 & 2 \\
\hline Parainfluenza virus & 1 & & 6 \\
\hline Rhinovirus & 1 & & 2 \\
\hline Adenovirus & & & 2 \\
\hline $\begin{array}{l}\text { Lower respiratory tract viral } \\
\text { infections } N=28(4 \%)\end{array}$ & $5(18)$ & $3(11)$ & $20(71)$ \\
\hline $\begin{array}{l}\text { Median time of LRT infections } \\
\text { days or months (range) }\end{array}$ & 17 days (19-22) & - & 7 months $(3-37)$ \\
\hline Respiratory syncytial virus & 2 & & 8 \\
\hline Influenza virus & 1 & 1 & 6 \\
\hline Parainfluenza virus & & 1 & 1 \\
\hline Coronavirus & 1 & & 1 \\
\hline Rhinovirus & 1 & & 3 \\
\hline Metapneumovirus & & 1 & 1 \\
\hline $\begin{array}{l}\text { Other viral infections } N=19 \\
(3 \%)\end{array}$ & $5(26)$ & $5(26)$ & $9(48)$ \\
\hline $\begin{array}{l}\text { Median time of other virus } \\
\text { infections days or months (range) }\end{array}$ & 15 days $(5-21)$ & 63 days (40-93) & 5,5 months $(3-12)$ \\
\hline Human herpes type 6 virus & 1 & 3 & 3 \\
\hline Adenovirus & 3 & & 1 \\
\hline Rotavirus & & & 2 \\
\hline Herpes simplex virus & & 2 & \\
\hline Hepes zoster virus & & & 2 \\
\hline Enterovirus & & & 1 \\
\hline Norovirus & 1 & & \\
\hline $\begin{array}{l}\text { Invasive fungal infections } N=41 \\
(7 \%)\end{array}$ & $14(34)$ & $13(32)$ & $14(34)$ \\
\hline $\begin{array}{l}\text { Median time of fungal infections } \\
\text { days or months (range) }\end{array}$ & 10 days $(0-28)$ & 72 day (31-99) & 11 months $(4-46)$ \\
\hline Possible IA & 2 & 2 & 2 \\
\hline Probable IA & 5 & 1 & 2 \\
\hline Proven IA & 1 & 3 & 4 \\
\hline Pneumocystis jirovecci pneumonia & & & 2 \\
\hline $\begin{array}{l}\text { Candida spp uncomplicated } \\
\text { fungemia }\end{array}$ & 6 & 7 & 3 \\
\hline Penicillinum spp & & & 1 \\
\hline
\end{tabular}

$G P B$ gram positive bacteria, $G N B$ gram negative bacteria, $C D$ clostridium difficile, $C M V$ cytomegalovirus, EBV-PTLD EBV-related Posttransplant lymphoproliferative disease, $H C$ Hemorrhagic cystitis, $U R T$ upper respirtory tract, $L R T$ lower respiratory tract, $I A$ invasive aspergillosis.

aureus (MRSA) and MR S. epidermidis, with 4 infections each). All GPB infections were BSI except for 3 cases of listeriosis and one case of nocardiosis.

There were 7 cases of IRM caused by GPB infections ( $16 \%$ of IRM), two infections before +30 days due to
Enterococcus faecium, three in the post-engraftment period caused by Streptococcus mitis, Enterococcus faecalis, and MRSA and two late infections $(>+100$ days) due to Enterococcus faecalis and Streptococcus pneumoniae (shown in detail in Table 3). 
With respect to infections by gram-negative bacteria $(\mathrm{GNB})$, the incidence of PE $(<$ day +31$)$, early postengraftment (day +31 to +100$)$ and late post-engraftment $(>$ day +100$)$ infections was $10 \%$ (95\% C.I., 6-14\%), $14 \%$ (95\% C.I., 9-19\%) and 22\% (95\% C.I., 16-28\%), respectively, with an overall incidence of $46 \%$. The median time of infection was 13 days (range 0-28), 51 days (range 35-97), and 7 months (range 3-33). Details are shown in Table 4. One hundred seven GNB infections occurred, all were BSI, and as apposed to GPB infections the highest rate of GNB infections occurred in the late post-engraftment period risk, with 50 episodes $(47 \%)$ of GNB infections. The most common pathogens isolated were Escherichia coli, Pseudomonas aeruginosa, and Klebsiella pneumoniae, which accounted for 83/107 (78\%) of GNB infections among these three species, $16 \%$ were antibiotic-resistant (eight multidrug-resistant (MDR) Pseudomonas aeruginosa, three extended-spectrum beta-lactamase producing (ESBL) Escherichia Coli and seven ESBL or carbapenamase producing Klebsiella pneumoniae). Other innately MDR species included 7 infections by Stenotrophomonas maltophila and one case of Acinetobacter baumanii.

The causes of IRM by GNB are shown in detail in Table 3, which accounted for 30\% of IRM (13 patients); two lethal infections in the PE period (MDR Pseudomonas aeruginosa and ESBL Escherichia coli), two in the early post-engraftment period (MDR Pseudomonas aeruginosa, and ESBL Klebsiella pneumonia), and 9 patients in the late post-engraftment period (one Pseudomonas aeruginosa and one MDR Pseudomonas aeruginosa; one ESBL Klebsiella pneumoniae and two Klebsiella pneumoniae, one Escherichia coli, one Serratia marcescense, Stenotrophomona maltophilia and Acinetobacter baumannii).

\section{Virus infections}

\section{CMV and EBV Infections}

The incidence of CMV infection (CMV-I) in PE period $(<$ day +31$)$ and post-engraftment period ( $\geq 31$ days) was $25 \%$ (95\% CI, 20-30\%) and 44\% (95\% CI, 38-50\%), respectively. In three patients were showed the CMV infection after 12 months. Among CMV seropositive patients the incidence of CMV-I was $78 \%$ (95\% C.I., 72-84\%), whereas it was $24 \%$ (95\% C.I., 12-36\%) in seronegative recipients. Five patients developed CMV pneumonitis, in four cases before day +31 and one on day +155 , and four died from this complication.

The incidence of EBV reactivation was $10 \%(95 \% \mathrm{CI}$, $6-14 \%)$, mainly in the late post-engraftment period $(18 / 23$ cases). Fifteen patients were treated with rituximab due to high EBV DNAemia ( $>1000$ e.g.c./mL). EBV-related posttransplant lymphoproliferative disorder (EBV-PTLD) was diagnosed in two patients and was lethal in one of these cases.
Community/conventional respiratory virus (CRV) infections

Sixty-two episodes of CRV infections were reported, and the incidence of at least one episode of CRV infection was $4 \%$ (95\% C.I., 1-7\%) in the PE period, 5\% (95\% C.I., 2-8\%) in the early post-engraftment and $15 \%$ (95\% C.I., 10-20\%) in the late post-engraftment period. Only one patient died as a consequence of metapneumovirus pneumonia.

Upper (URTI) and lower (LRTI) respiratory tract infections by a CRV accounted for 34 and 28 episodes (55\% and 45\%), respectively (details shown in Table 4). Both URTI and LRTI occurred mostly in the late post-engraftment period (65 and $71 \%$ of CRV infections, respectively). The most CRV in URTI were influenza viruses (14 episodes), respiratory sincytial virus (7 episodes) and parainfluenza viruses (7 episodes), whereas respiratory sincytial virus (10 episodes) and influenza viruses (6 episodes) were the most common in LRTI.

\section{Other viral infections}

A very frequent complication in haploSCT protocols with PTCy is hemorrhagic cystitis (HC), as confirmed in the current study. The incidence of $\mathrm{HC}$ until +30 days was $14 \%(95 \% \mathrm{C}$. I., $10-18 \%)$ and after day +31 it was $21 \%$ (95\% C.I., 16-26\%), for an overall incidence of 35\%. BK-polyomavirusrelated $\mathrm{HC}$ was diagnosed in 54 cases $(70 \%$ of $\mathrm{HC}$ ), while adenovirus-related $\mathrm{HC}$ was found in five cases (6\% of $\mathrm{HC}$ ). No viral pathogen was identified in 18/77 cases of HC (23\%), 11 of which occurred early post-transplant.

Human Herpes virus type 6 infection was diagnosed in 7 patients, with two cases of encephalitis and 5 cases of colitis. Other less common cutaneous and intestinal viral infections are shown in Table 4.

\section{Invasive fungal infections}

The incidence of invasive fungal infections (IFI) was $4 \%$ (95\% C.I., 3-5\%) before +31 days and $7 \%$ (95\% C.I., $4-10 \%$ ) after day +31 , for a 3 -year incidence of $11 \%$. The most common IFI was invasive aspergillosis (IA), with eight cases of proven IA, eight probable IA and six cases of possible IA. An IFI was the primary cause of death for two patients with proven IA and one probable IA. Pneumocystis jirovecci pneumonia was diagnosed in the late post engraftment period in only two patients, while uncomplicated candidemia occurred in 16 patients $(7 \%)$ [details in Table 4].

\section{Discussion}

In the current study, we describe the incidence of severe infections and the IRM in a large series of adult recipients of a haploSCT with PTCy. As previously described in other 
studies infectious complications are the main cause of NRM, as occurs with other types of alloSCT $[25,26]$. The 3 -year incidence of NRM was $31 \%$ and $19 \%$ for IRM in the present study.

In our series, the 3-year incidence of GPB and GNB infections was $51 \%$ and $46 \%$, respectively (246 episodes), similar to the incidence reported by others which range from $35 \%$ to $62 \%$ [27-30], albeit somewhat different definitions were used in different studies. The rates of GPB infections were similar in the pre-engraftment and late post-engraftment period, while GNB infections were more common in the late post-engraftment period ( $>$ day $+100)$. Risk factors for these late GNB infections have been reported, although we did not analyze their risk factors due to the large number of species involved and thus small numbers per pathogen [14]. Bacterial infections were the most common causes of IRM (51\%, 22 patients), mainly GNB infections (30\%, 13 patients). As expected, Escherichia coli, Pseudomonas aeruginosa, and Klebsiella pneumoniae were the most common GNB species [14, 27, 29, 31].

Antibiotic resistance appeared to contribute to IRM. A MDR strain was involved in 6/13 (46\%) deaths by GNB, while only 16/83 (19\%) overall isolated were MDR, and three additional patients died due to Acinetobacter baumannii, Serratia marcescens, and Stenotrophomonas maltophilia. Knowledge of the epidemiology of MDR GNB in each hospital as well as prior infections by these pathogens in any given patient is crucial in establishing the best empirical antibiotic strategy [14, 32, 33]. On the other hand, a high incidence of GPB was found during the study; however, antibiotic resistance was rare among GPB (6\%), and only one patient died due to MRSA infection.

Regarding CMV-I, a high incidence was found in our series ( $24 \%$ during PE period and $45 \%$ beyond $\geq 31$ days), in contrast to a low rate of CMV disease (2\%), as reported by others [27, 29, 34-36]. A higher incidence of CMV-I in haploSCT with PTCy compared with other donor types has been reported previously [27, 36-38]. Initial studies hypothesized that the high incidence of CMV-I correlated with delayed CD4 + T cell and dendritic cell recovery [39, 40]. Also, CMV-I has a strong impact on the integrity and heterogeneity of the $\mathrm{T}$ cell repertoire, leading to $\mathrm{CD} 8+$ effector memory $\mathrm{T}$ cell expansion and contraction of naïve cells [41]. However, a recent report found that CMV-specific-T-cell reconstitution in T-cell replete haploSCT with PTCy was comparable to other types of alloSCT without PTCy [42]. More studies are necessary to define the relationship between CMV-I and immunological reconstitution.

Interestingly, we found a low incidence of EBV-I (10\%) and EBV-PTLD (2 patients), as recently reported by other groups $[27,43]$. The immunological hypothesis for the low incidence of EBV-I and EBV-PTLD is unclear, but the lack of in vivo or ex vivo T-cell depletion is of course a major determinant for the low incidence [42].

In haploSCT with PTCy the incidence of $\mathrm{HC}$ has been reported to range from $19 \%$ to $60 \%[38,44]$. Recent publications showed a higher incidence of HC in haploSCT with PTCy than in alloSCT from matched related donors also with PTCy (55\% vs. $25 \%$ ) [44, 45], suggesting that the use of PTCy is not the main risk factor for the higher incidence of $\mathrm{HC}$ in haploSCT. As expected, BK Polyomavirus was the most common virus linked to $\mathrm{HC}$ (70\% of cases) in our series. In the haploSCT setting donor T lymphocytes are HLA mismatched with urothelial viral antigenpresenting cells, compromising the immune effector $\mathrm{T}$ cell response [45]. Although there is no treatment nor prophylaxis for viral-related $\mathrm{HC}$, the continuous intravenous infusion of MESNA has been recently reported to reduce the incidence of $\mathrm{HC}$ when compared with bolus administration (5.6\% vs. $27.8 \%$ ) [46], although this requires confirmation with further studies.

A low incidence of IFI was found (11\% at 3 years), especially during the early aplastic post-transplant period (4\%) and with a very low impact on IRM. Due to the low incidence, we were unable to analyze the risk factors for developing an IFI.

Among the patients on immunosuppressive drugs (IS) during the entire study follow-up, $40 \%$ of all IRM (17 patients) occurred in patients after +100 days and $44 \%$ of all IRM occurred before day +100 (19 patients). However, in the 161 patients who discontinued IS post-transplant the IRM was low (7 patients, $4.3 \%$ rate of and $16 \%$ of all the cases of IRM).

The present study shares the limitations inherent to retrospective studies, including potential selection bias; and the uncertainty of whether all infections were captured and included in the study. However, the study gives a useful picture of the overall epidemiology of different severe infections and their impact on IRM in the setting of adult haploSCT with PTCy in our country.

In conclusion, our national study shows that IRM is the main cause of NRM in the haploSCT setting with PT-Cy. A major cause of IRM were GNB infections, possibly higher in patients with MDR GNB infections. Studies focusing on the immunological reconstitution, especially in patients without severe GVHD, may help in understanding the high incidence of late infections linked to cellular immunity, such as CMV and viral-related $\mathrm{HC}$.

Funding This project has been funded by La Marató de TV3 Foundation file number: 20133230. AGAUR 2017 SGR 1395 from the Catalan Government and a grant from "Obra Social La Caixa" Barcelona Spain. PERIS SLT002/16/0043 from the Catalan Government and FIS PI17/ 01246 from the Instituto de Salud Carlos III, Ministerio de Economía y Competitividad, Spain. Tercel RD16/0011/0028 Instituto de Salud Carlos III, Ministerio de Economía y Competitividad, Spain. 


\section{Compliance with ethical standards}

Conflict of interest The authors declare no competing interest.

Publisher's note Springer Nature remains neutral with regard to jurisdictional claims in published maps and institutional affiliations.

\section{References}

1. Aversa F, Terenzi A, Tabilio A, Falzetti F, Carotti A, Ballanti S, et al. Full haplotype-mismatched hematopoietic stem-cell transplantation: a phase II study in patients with acute leukemia at high risk of relapse. J Clin Oncol. 2005;23:3447-54.

2. Federmann B, Bornhauser M, Meisner C, Kordelas L, Beelen DW, Stuhler G, et al. Haploidentical allogeneic hematopoietic cell transplantation in adults using CD3/CD19 depletion and reduced intensity conditioning: a phase II study. Haematologica. 2012;97:1523-31.

3. Huang XJ, Liu DH, Liu KY, Xu LP, Chen H, Han W, et al. Haploidentical hematopoietic stem cell transplantation without in vitro T-cell depletion for the treatment of hematological malignancies. Bone Marrow Transpl. 2006;38:291-7.

4. Luznik L, O'Donnell P, Symons H, Chen AR, Leffell MS, Zahurak M, et al. HLA haploidentical bone marrow transplantation for hematologic malignancies using nonmyeloablative conditioning and high-dose, posttransplantation cyclophosphamide. Biol Blood Marrow Transpl. 2008;14:641-50.

5. Raiola AM, Dominietto A, di Grazia C, Lamparelli T, Gualandi F, Ibatici A, et al. Unmanipulated haploidentical transplants compared with other alternative donors and matched sibling grafts. Biol Blood Marrow Transpl. 2014;20:1573e1579.

6. Ruggeri A, Labopin M, Sanz G, Piemontese S, Arcese W, Bacigalupo A, et al. Comparison of outcomes after unrelated cord blood and unmanipulated haploidentical stem cell transplantation in adults with acute leukemia. Leukemia 2015;29:1891-900.

7. Ciurea SO, Zhang M-J, Bacigalupo AA, Bashey A, Appelbaum FR, Aljitawi OS, et al. Haploidentical transplant with posttransplant cyclophosphamide vs matched unrelated donor transplant for acute myeloid leukemia. Blood 2015;126:1033-40.

8. Kwon M, Bautista G, Balsalobre P, Sanchez Ortega I, Montesino P, Bermudez A, et al. Haplo-cord transplantation compared to haploidentical transplantation with post-transplant cyclophosphamide in patients with AML. Bone Marrow Transpl. 2017;52:1138-43.

9. Bashey A, Solomon SR. T-cell replete haploidentical donor transplantation using post-transplant CY: an emerging standardof-care option for patients who lack an HLA-identical sibling donor. Bone Marrow Transpl. 2014;49:999-1008.

10. Brissot E, Labopin M, Ehninger G, Stelljes M, Brecht A, Ganser A, et al. Haploidentical versus unrelated allogeneic stem cell transplantation for relapsed/refractory acute myeloid leukemia: a report on 1578 patients from the Acute Leukemia Working Party of the EBMT. Haematologica 2019;104:524-32.

11. Bashey A, Zhang A, Jackson K, Brown S, Ridgeway M, Solh M, et al. Comparison of outcomes of hematopoietic cell transplants from T-replete haploidentical donors using post-transplantation cyclophosphamide with 10 of 10 HLA-A, -B, -C, -DRB1, and -DQB1 allele-matched unrelated donors and HLA-identical sibling donors: a multivariable analysis including disease risk index. Biol Blood Marrow Transpl. 2016;22:125-33.

12. Atilla E, Atilla PA, Bozdağ SC, Demirer T. A review of infectious complications after haploidentical hematopoietic stem cell transplantations. Infection. 2017;45:403-11.

13. Yan $\mathrm{CH}$, Wang $\mathrm{Y}$, Mo XD, Sun $\mathrm{YQ}$, Wang FR, Fu H, et al. Incidence, risk factors, microbiology and outcomes of pre-engraftment bloodstream infection after haploidentical hematopoietic stem cell transplantation and comparison with HLA-identical sibling transplantation. Clin Infect Dis. 2018;67:S162-S173.

14. Girmenia C, Bertaina A, Piciocchi A, Perruccio K, Algarotti A, Busca A, et al. Incidence, risk factors and outcome of preengraftment gram-negative bacteremia after allogeneic and autologous hematopoietic stem cell transplantation: an Italian Prospective Multicenter Survey. Clin Infect Dis. 2017;65:1884-96.

15. Herbers A, Haan A, van der Velden W, Donnelly J, Blijlevens N. Mucositis not neutropenia determines bacteremia among hematopoietic stem cell transplant recipients. Transpl Infect Dis. 2014;16:279-85.

16. Modi A, Rybicki L, Majhail NS, Mossad SB. Severity of acute gastrointestinal graft-vs-host disease is associated with incidence of bloodstream infection after adult allogeneic hematopoietic stem cell transplantation. Transpl Infect Dis. 2020;22:e13217.

17. Aversa F, Tabilio A, Velardi A, Cunningham I, Terenzi A, Falzetti F, et al. Treatment of high-risk acute leukemia with T-celldepleted stem cells from related donors with one fully mismatched HLA haplotype. N. Engl J Med. 1998;339:1186-9.

18. Luznik L, O'Donnell PV, Fuchs EJ. Post-transplantation cyclophosphamide for tolerance induction in HLA-haploidentical bone marrow transplantation. Semin Oncol. 2012;39:683-93.

19. Kanakry CG, Fuchs EJ, Luznik L. Modern approaches to HLA haploidentical blood or marrow transplantation. Nat Rev Clin Oncol. 2016;13:10-24.

20. Raiola AM, Dominietto A, Ghiso A, Di Grazia C, Lamparelli T, Gualandi $F$, et al. Unmanipulated haploidentical bone marrow transplantation and posttransplantation cyclophosphamide for hematologic malignancies after myeloablative conditioning. Biol Blood Marrow Transpl. 2013;19:117-22.

21. Arcese W, Cerretti R, Sarmati L, Cudillo L, De Angelis G, Mariotti B, et al. Matched-pair analysis of transplant from haploidentical, unmanipulated bone marrow donor versus HLA identical sibling for patients with hematologic malignancies. Biol Blood Marrow Transpl. 2020;S1083-8791:30089-6.

22. Gayoso J, Balsalobre P, Kwon M, Herrera P, Bermúdez A, Sampol $\mathrm{A}$, et al. Busulfan based myeloablative conditioning regimens for haploidentical transplantation in high risk acute leukemias and myelodysplastic syndromes. Eur J Haematol. 2018;101:332-9.

23. Esquirol A, Pascual MJ, Ortiz M, Piñana JL, Ferra C, Garcia Cadenas I, et al. Single-agent GvHD prophylaxis with tacrolimus after post-transplant high-dose cyclophosphamide is a valid option for haploidentical transplantation in adults with hematological malignancies. Bone Marrow Transpl. 2017;52:1273-9.

24. Armand P, Kim HT, Logan BR, Wang Z, Alyea EP, Kalaycio ME, et al. Validation and refinement of the Disease Risk Index for allogeneic stem cell transplantation. Blood 2014;123:3664-71.

25. Ullmann AJ, Schmidt-Hieber M, Bertz H, Heinz WJ, Kiehl M, Krüger W, et al. Infectious diseases in allogeneic haematopoietic stem cell transplantation: prevention and prophylaxis strategy guidelines 2016. Ann Hematol. 2016;95:1435-55.

26. Forcina A, Lorentino F, Marasco V, Oltolini C, Marcatti M, Greco $\mathrm{R}$, et al. Clinical Impact of Pretransplant Multidrug-Resistant Gram-Negative Colonization in Autologous and Allogeneic Hematopoietic Stem Cell Transplantation. Biol Blood Marrow Transpl. 2018;24:1476-82.

27. Crocchiolo R, Bramanti S, Vai A, Sarina B, Mineri R, Casari E, et al. Infections after T-replete haploidentical transplantation and high-dose cyclophosphamide as graft-versus-host disease prophylaxis. Transpl Infect Dis. 2015;17:242-9.

28. Fayard A, Daguenet E, Blaise D, Chevallier P, Labussière H, Berceanu A, et al. Evaluation of infectious complications after haploidentical hematopoietic stem cell transplantation with posttransplant cyclophosphamide following reduced-intensity and myeloablative conditioning: a study on behalf of the francophone 
society of stem cell transplantation and cellular therapy (SFGMTC). Bone Marrow Transpl. 2019;54:1586-94.

29. Slade M, Goldsmith S, Romee R, DiPersio JF, Dubberke ER, Westervelt $\mathrm{P}$, et al. Epidemiology of infections following haploidentical peripheral blood hematopoietic cell transplantation. Transpl Infect Dis. 2017;19:e12629.

30. Oltolini C, Greco R, Galli L, Clerici D, Lorentino F, Xue E, et al. Infections after allogenic transplant with post-transplant cyclophosphamide: impact of donor HLA-matching. Biol Blood Marrow Transpl. 2020;26:1179-88.

31. Averbuch D, Tridello G, Hoek J, Mikulska M, Akan H, Yanez L, et al. Antimicrobial Resistance in Gram-Negative Rods Causing Bacteremia in Hematopoietic Stem Cell Transplant Recipients: Intercontinental Prospective Study of the Infectious Diseases Working Party of the European Bone Marrow Transplantation Group. Clin Infect Dis. 2017;65(11):1819-28.

32. Scheich S, Lindner S, Koenig R, Reinheimer C, Wichelhaus TA, Hogardt M, et al. Clinical impact of colonization with multidrugresistant organisms on outcome after allogeneic stem cell transplantation in patients with acute myeloid leukemia. Cancer 2018; 124:286-96.

33. Goldsmith SR, Slade M, DiPersio JF, Westervelt P, Lawrence SJ, Uy GL, et al. Cytomegalovirus viremia, disease, and impact on relapse in T-cell replete peripheral blood haploidentical hematopoietic cell transplantation with post-transplant cyclophosphamide. Haematologica 2016;101:e465-e468.

34. Lin CH, Su YJ, Hsu CY, Wang PN, Teng CJ. Haploidentical allogeneic hematopoietic stem cell transplantation increases the risk of cytomegalovirus infection in adult patients with acute leukemia. Transpl Infect Dis. 2019;20:e13096.

35. Huntley D, Giménez E, Pascual MJ, Hernández-Boluda JC, Gago B, Vázquez L, et al. Incidence, features, and outcomes of cytomegalovirus DNAemia in unmanipulated haploidentical allogeneic hematopoietic stem cell transplantation with post-transplantation cyclophosphamide. Transpl Infect Dis. 2020;22:e13206.

36. Stasi A, Milton DR, Poon LM, Hamdi A, Rondon G, Chen J, et al. Similar transplant outcomes for AML/MDS patients with haploidentical versus 10/10 HLA matched unrelated and related donors. Biol Blood Marrow Transpl. 2014;20:1975-81.
37. Tischer J, Engel $\mathrm{N}$, Fritsch $\mathrm{S}$, Prevalsek D, Hubmann $\mathrm{M}$, Schulz C, et al. Virus infection in HLA-haploidentical hematopoietic stem cell transplantation: incidence in the context of immune recovery in two different transplantation settings. Ann Hematol. 2015;94:1677-88.

38. McCurdy SR, Luznik L. Immune reconstitution after T-cell replete HLA-haploidentical transplantation. Semin Hematol. 2019;56:221-6.

39. Chang YJ, Zhao XY, Huang XJ. Immune reconstitution after haploidentical hematopoietic stem cell transplantation. Biol Blood Marrow Transpl. 2014;20:440-9.

40. Suessmuth Y, Mukherjee R, Watkins B, Koura DT, Finstermeier $\mathrm{K}$, Desmarais $\mathrm{C}$, et al. CMV reactivation drives post-transplant $\mathrm{T}$ cell reconstitution and results in defects in the underlying TCR $\beta$ repertoire. Blood. 2015;125:3835-50.

41. Huntley D, Giménez E, Pascual MJ, Remigia MJ, Amat P, Vazquez L, et al. Reconstitution of cytomegalovirus-specific Tcell immunity following unmanipulated haploidentical allogeneic hematopoietic stem cell transplantation with posttransplant cyclophosphamide. Bone Marrow Transplant. 2020;55:1347-56.

42. Kanakry JA, Kasamon YL, Bolaños-Meade J, Borrello IM, Brodsky RA, Fuchs E, et al. Absence of posttransplantation lymphoproliferative disorder after allogeneic blood or marrow transplantation using posttransplantation cyclophosphamide as graft-versus-host disease prophylaxis. Biol Blood Marrow Transpl. 2013;19(10): 1514-7.

43. Lunde LE, Dasaraju S, Cao Q, Cohn CS, Reding M, Bejanyan N, et al. Hemorrhagic cystitis after allogeneic hematopoietic cell transplantation: risk factors, graft source and survival. Bone Marrow Transpl. 2015;50:1432-7.

44. Copelan OR, Sanikommu SR, Trivedi JS, Butler C, Ai J, Ragon $\mathrm{BK}$, et al. Higher incidence of hemorrhagic cystitis following haploidentical related donor transplantation compared with matched related donor transplantation. Biol Blood Marrow Transpl. 2019;25:785-90.

45. Arango M, Cardona D. Hemorrhagic cystitis after haploidentical transplantation with post-transplant cyclophosphamide: protective effect of MESNA continuous infusion. Biol Blood Marrow Transpl. 2020;26(8):1492-6. 